\title{
Real-Time Fusion of Ultrasound and Gamma Probe for Navigated Localization of Liver Metastases
}

\author{
Thomas Wendler ${ }^{1}$, Marco Feuerstein ${ }^{1}$, Joerg Traub ${ }^{1}$, Tobias Lasser ${ }^{1}$, \\ Jakob Vogel ${ }^{1}$, Farhad Daghighian ${ }^{2}$, Sibylle I. Ziegler ${ }^{3}$, and Nassir Navab ${ }^{1}$ \\ ${ }^{1}$ Computer Aided Medical Procedures (CAMP), TUM, Munich, Germany \\ ${ }^{2}$ IntraMedical Imaging LLC, Los Angeles, California, USA \\ 3 Nuclear Medicine Department, Klinikum rechts der Isar, TUM, Munich Germany
}

\begin{abstract}
Liver metastases are an advanced stage of several types of cancer, usually treated with surgery. Intra-operative localization of these lesions is currently facilitated by intra-operative ultrasound (IOUS) and palpation, yielding a high rate of false positives due to benign abnormal regions. In this paper we present the integration of functional nuclear information from a gamma probe with IOUS, to provide a synchronized, real-time visualization that facilitates the detection of active metastases intra-operatively. We evaluate the system in an ex-vivo setup employing a group of physicians and medical technicians and show that the addition of functional imaging improves the accuracy of localizing and identifying malignant and benign lesions significantly. Furthermore we are able to demonstrate that the inclusion of an advanced, augmented visualization provides more reliability and confidence on classifying these lesions in the presented evaluation setup.
\end{abstract}

\section{Motivation}

Liver metastases are a common consequence of cancer cells spreading from primary tumors. Surgical resection is the indicated therapy if possible, as it results in a cure with high probability [1]. To facilitate extraction, intra-operative localization of the tumorous regions is achieved by a combination of palpation and intra-operative ultrasound (IOUS). This technique is considered the gold standard as it has been in successful clinical practice for years already with a proved high sensitivity [2,3]. However, in the presence of benign abnormal structures, a considerable false-positive detection rate still remains. These abnormalities may be cysts, hemangiomas, scar tissue, or even metastases, which were previously diagnosed by e.g. PET/CT and treated successfully with chemotherapy or other neoadjuvant therapies 3 . This problem, although reduced, is still present when using contrast-enhanced ultrasound [2, which has a promising potential for better image quality, but still remains a mostly anatomical imaging modality.

To reduce the detection rate of false-positives, the integration of a functional modality to complement the standard localization technique is a promising approach. A prime candidate for this is nuclear imaging, as there are tracers with 
high specific uptake in liver metastases 4], and in general this modality features low false-positive detection rates [5] in pre-operative diagnostics.

Herein we report on combining an intra-operative nuclear probe (a gamma probe) with ultrasound (IOUS) for accurate localization of liver metastases. We believe that this combination will result in an excellent compensation of the weak sides of both technologies, achieving accurate localization and accurate classification. In order to take full advantage of this combination the relative position and orientation of both ultrasound probe and nuclear probe need to be known to allow a merged visualization of both functional gamma probe information and anatomical ultrasound images and thus a complete utilization of both technologies.

\section{System Setup}

The components of a combined IOUS / nuclear probe system for the detection of liver metastases, their calibration and a visualization to intuitively guide surgeons are reviewed briefly in this section.

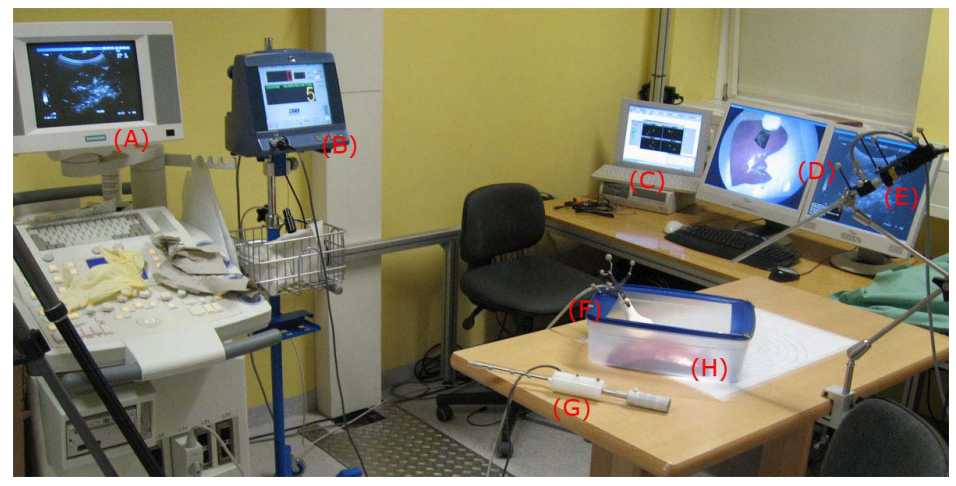

Fig. 1. The system setup of the navigation system consists of a US system (A), a gamma probe system (B), and a tracking system (C). The laparoscopic camera (E), the US probe $(\mathrm{F})$, and the gamma probe $(\mathrm{G})$ are extended by targets for optical tracking. Radioactive and non-radioactive nodules have been implanted in the liver $(\mathrm{H})$. The navigation system provides different modalities of visualization (D).

\subsection{System Components}

Tracking System. The determination of position and orientation of ultrasound and nuclear probes in a common coordinate system can be achieved by an optical tracking system, as in our previous work, where we track a beta-probe optically and generate the radioactivity surface distribution of the resection borders of a tumor 6]. A similar approach is used in this work. Both nuclear and IOUS probes are extended by infrared tracking targets (figure 1). The chosen optical tracking system (A.R.T. GmbH, Weilheim, Germany) consists of 4 ARTtrack2 
infrared cameras and the DTrack software running on a desktop PC, which sends tracking data via Ethernet to the central workstation. A typical setup with four ART cameras has a root mean square (rms) error of $0.4[\mathrm{~mm}]$ for the target position and $0.002[\mathrm{rad}]$ for the target orientation and a maximum error of $1.4[\mathrm{~mm}]$ for positional and $0.007[\mathrm{rad}]$ for orientation measurements.

Ultrasound. In order to obtain 2D anatomical images of the liver in real-time, an ultrasound probe of $3.5[\mathrm{MHz}]$ is connected to a SonolineOmnia system by Siemens Medical Solutions (Mountain View, California, USA). Its B-scan images are captured by a frame-grabber card in the central workstation.

Gamma Probe. The equipment to detect the radioactive tracers is a standard gamma probe attached to a NodeSeeker control unit, both by IntraMedical Imaging LLC (Los Angeles, California). The gamma probe is equipped with a custom-made, external collimator to reduce its field of view (FOV) to a cone of $0.1[\mathrm{rad}]$ opening. This value was chosen to allow a small FOV while still preserving a reasonable sensitivity to the chosen radiation. The measured sensitivity is $0.75[c p s / k B q]$ for a point source of Tc-99m located on the axis of the probe at a distance of $5[\mathrm{~cm}]$. The energy window was set to $20 \%$ centered at $140[\mathrm{keV}]$ ideally for Tc-99m.

\subsection{System Calibration}

Ultrasound. For US calibration, i.e. the determination of the US plane's pixel size and the rigid transformation from US plane to the tracking target, we adopted the single-wall calibration method of Prager et al. [7, using a nylon membrane as proposed by Lang $\varnothing$ [8]. Several positions and orientations of the probe's tracking target as well as corresponding images of the membrane inside a water bath are acquired synchronously. The lines corresponding to the planar membrane are automatically segmented and used for the computation of all calibration parameters. As suggested by Treece et al. [9] we determine the temporal offset between US acquisition and tracking for better data synchronization. Furthermore, we adopted their calibration protocol to ensure numeric stability for all degrees of freedom of the transformation. To determine the US calibration accuracy, a tracked pointer with tip coordinates given in the tracking coordinate system was submerged into the water bath. Its tip was segmented manually in 5 regions of the US plane, which was repeated for 4 poses of the probe differing from the ones used during calibration. The pointer tip's coordinates were transformed into the US plane coordinate system and compared to the segmented tip coordinates (scaled to millimeters). An rms error of $1.17[\mathrm{~mm}] \pm 0.40[\mathrm{~mm}]$ and a maximum error of $1.58[\mathrm{~mm}]$ were obtained.

Gamma Probe. The calibration of the nuclear probe consists of two steps. First, the rigid transformation from the tracking target to the sensor (scintillator crystal) is determined. As the probe is symmetric along the central axis, it can be exploited by designing a tracking target along this axis (figure 1). Thus the 


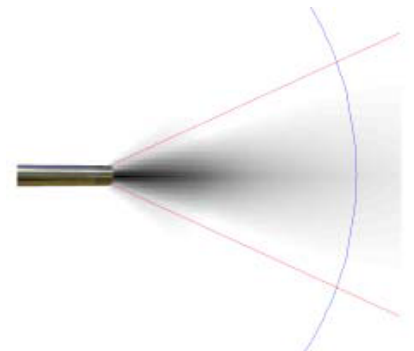

(a) Gamma probe calibration.

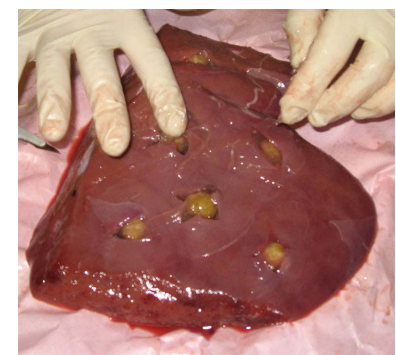

(b) Preparation of phantom.

Fig. 2. (a) Schematic representation of the FOV of the gamma probe. The background color represents the measured sensitivity of the probe at the different positions (the darker the higher the sensitivity). The red and blue lines define the angle of aperture and the height of the cone respectively. (b) Liver used for experiments. The wax spheres were implanted before the experiment, some of them were radioactive. During the experiment test persons mark the detected hot nodules in the liver with needles.

problem reduces to determine the distance between sensor and origin of the tracking target, which can be fixed by construction. In a second step, the FOV of the probe is measured, which can be approximated by a cone. The main calibration task is to determine the height of the cone and its angle of aperture. Therefor, a point source of radioactivity is placed at known relative distances and angles from the sensor and the mean count rate is stored. Hence both the maximum distance and maximum angle to detect a significant count rate are determined (figure 2(a). The criterion chosen was to have a count rate at least 2.5 times higher than the local background, which is commonly used to identify lesions in nuclear medicine [10.

\subsection{Visualization}

The visualization comprises an augmentation of nuclear information on the IOUS image (figure $3(\mathrm{a})$ as well as a $3 \mathrm{D}$ visualization of the relative position and orientation of both probes in an augmented reality mode (figure 3(b) . In both cases a proper synchronization is needed. For this purpose we have used and extended a framework capable of integrating and synchronizing several data streams for augmented reality applications [1].

For the overlay of nuclear information on the B-mode IOUS plane, the intersection of the plane with the FOV of the nuclear probe needs to be calculated. This can be achieved by intersecting the quadric that represents the FOV of the nuclear probe (in this case a cone) with the plane of the IOUS, resulting in a conic section. Its parameters are calculated, and whenever the conic section is closed (i.e. its eccentricity $<1$ ), it is augmented on the IOUS image (figure $3(\mathrm{a})$. This is done in real-time using the tracking system to acquire the pose of both, the axis of the nuclear probe and the plane of the IOUS probe. The color of the ellipse of intersection is dependent on the nuclear readings. It is visualized 


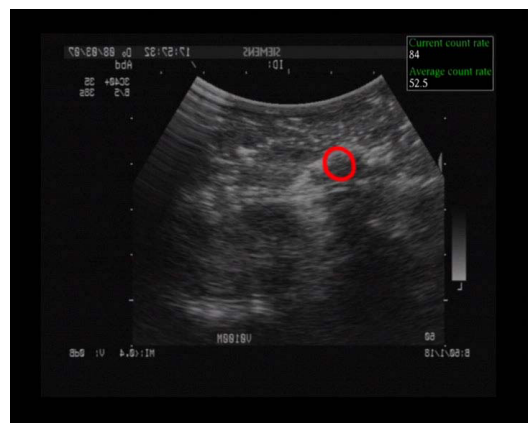

(a) Augmented IOUS plane.

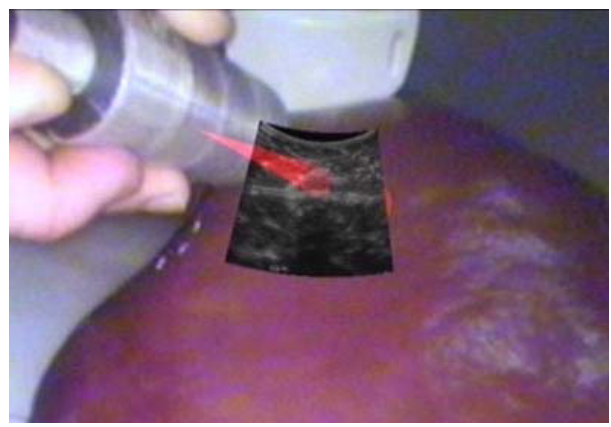

(b) Augmented reality view.

Fig. 3. Different visualization modes of the navigation

in red when the reading is above 2.5 times the background, otherwise it is drawn in green.

The $3 \mathrm{D}$ visualization is achieved by projecting the IOUS plane and the cone that represents the FOV of the probe onto the image of an augmented camera. For this, a tracked calibrated camera (e.g. a laparoscope camera) is used (figure $3(\mathrm{~b})$. The color of the cone here is also encoded in red and green dependent on the nuclear activity, as described above for the conic section.

\section{Experiments}

For the experimental evaluation of the system a group of nuclear medicine doctors and technicians (4 each) was asked to find abnormalities in several cow livers using IOUS either exclusively or in combination with the nuclear probe. This setup simulates a clinical open liver metastasis resection. Radioactive and non-radioactive wax spheres [12] were implanted into the livers in advance, simulating malignant and benign metastases, respectively. The diameters of the wax 'tumors' varied from 7 to $9[\mathrm{~mm}]$. They were implanted from behind, so no scars were visible from top (figure 2(b)]. The concentration of the radioisotope $T c$ $99 \mathrm{~m}$ used to label 'malignant tumors' was $50[\mathrm{kBq} / \mathrm{ml}]$. In each liver 10 'lesions' were implanted, half of them being radioactive.

Once implanted with the wax 'lesions', the livers were scanned in a PET/CT device (Biograph16 PET/CT, Siemens Medical Solutions, Erlangen, Germany) to provide 'pre-operative' diagnostic data. Before giving form to the wax sphere $F-18$ at a concentration of $20[\mathrm{kBq} / \mathrm{ml}]$ was added to the radioactive wax mixture in order to enable detection by PET.

Three variants of lesion localization and classification were compared:

A) Use of pre-operative PET/CT images and IOUS.

B) Use of pre-operative PET/CT images and IOUS aided by gamma probe readings (no visualization). 
C) Use of pre-operative PET/CT images and IOUS aided by gamma probe readings employing the system described in section 2 for navigation of the probes and visualization.

The first method represents the current standard in clinical use, while the second and third method add a gamma probe as a second modality to improve accuracy and classification results. Method $\mathrm{C}$ also adds navigation and visualization to guide the test person and further improve the quality of the results.

After explaining how the modalities to be evaluated work and what was expected from them, the test persons were allowed to study the 'pre-operative' diagnostic PET/CT images. Then they were asked to localize the metastases in 3 differently marked livers, using one of the variants $\mathrm{A}, \mathrm{B}$ or $\mathrm{C}$ for each liver respectively. Palpation and further evaluation of the preoperative images during the procedure was allowed in all variants.

The parameters observed were the total amount of time needed to localize the metastases and the success of the localization in terms of positional accuracy, detection of abnormalities, and their characterization. Furthermore a questionnaire was filled out by each subject.

\section{Results}

On the one hand, a significant difference in the performance of variant A with respect to the gamma-aided variants $\mathrm{B}$ and $\mathrm{C}$ is shown (cf. table 1). On the other hand, the difference between the latter is not statistically relevant. Both gamma-aided variants achieve high sensitivity and specificity values $(97.5 \%$ to $100 \%$ both), whereas variant A presents significant amounts of false-positives and false-negatives (sensitivity $80.5 \%$, specificity $82.1 \%$ ). This result holds for doctors and technicians, the latter achieving slightly better results, which is probably due to the lack of expertise with the devices and thus a more thorough use of them (mirrored in time taken, see below).

Table 1. Performance evaluation (evaluated lesions)

\begin{tabular}{c||cc|cc|cc}
\multicolumn{1}{|||}{} & \multicolumn{2}{c|}{ A } & \multicolumn{2}{c|}{ B } & \multicolumn{2}{c}{ C } \\
& True False & True False & True False \\
\hline \hline Positive & 33 & 7 & 39 & 0 & 39 & 1 \\
Negative & 32 & 8 & 40 & 1 & 39 & 1
\end{tabular}

When analyzing the time needed for the task, the results show a clear trend: The non-integrated gamma-aided variant $B$ required $30 \pm 20 \%$ more time than the pure US variant A $(13.6 \pm 4.1[\mathrm{~min}])$, whereas the navigated variant took $70 \pm 30 \%$ longer.

Although both novel variants perform equally well statistically, the qualitative evaluation shows that the inclusion of navigation made the subjects feel more confident about their results $(3.0 \pm 0.7$ vs. $4.0 \pm 0.7$ and $4.4 \pm 0.5$ for variants $\mathrm{A}$, 
$\mathrm{B}$, and $\mathrm{C}$, respectively, on a scale from 1 to 5 for question 'Do you feel confident about the method?'; similar results for question 'Do you feel confident about the technology?'). These results were equally distributed for physicians and technical personnel. Effects of a learning curve were not evaluated and are part of current research.

A common opinion expressed by test persons was that without the aid of $\mathrm{PET} / \mathrm{CT}$ diagnostic data both non-navigated variants A and B would not allow precise localization and classification, in contrast to the navigated one $(\mathrm{C})$. This is of high interest, as for typical clinical cases the PET/CT images are often not recent but up to several weeks old. During this interval, anatomy may show big changes, especially if the metastases undergo treatment. Thus a more realistic test setup should consider changes in the malignancy of the metastases (i.e. the radioactivity concentration) and anatomical changes like the appearance of new disease foci. In that sense the performance for variants $\mathrm{A}$ and $\mathrm{B}$ has to be considered as the best case possible.

\section{Discussion}

Standard localization of liver metastases does not use real-time functional information during the procedure (beyond palpation). Including intra-operative nuclear probes into the procedure would slightly change the current workflow, increase costs (radionuclides), and introduce a small dose of radioactivity into the patient $(<1[m S v])$. However, these drawbacks should be compensated by the reduction of false-positives, as resection poses a bigger burden to patients.

In this implementation low-energy cancer tracers 3.5 are assumed since they require small and thus light collimators (for energies below $\approx 250[\mathrm{keV}]$ only a couple of millimeters of lead are sufficient for shielding). Unfortunately this leaves out the possibility of using the system with several successful cancer tracers like F-18-FDG, due to their high-energy nature (energy above $400[\mathrm{keV}]$, where some centimeters of lead are needed for effective shielding). However, new electronically collimated probes [13] are enabling the construction of light and handy devices with a small field of view for high-energy gamma detection, which will consequently allow the use of F-18-FDG and others in the near future.

The choice of patients that would qualify for this treatment is also an issue. Our current clinical studies show, that patients that present hot spots in preoperative images do also present intra-operatively. This is not valid viceversa, which would enable the use of the system in more patients. However, better selection criteria are yet not available.

Rigid mechanical coupling of the IOUS and gamma probes might serve as a simpler solution for the determination of the relative pose of both probes. The tracked variant however is more flexible and easy to handle, as it allows the surgeon to independently position the probes as desired and to have closer access to the patient's anatomy, which improves the quality of both ultrasound images and nuclear readings. Furthermore, as a next development step the proposed 
system will also be extended to laparoscopic probes, where mechanical coupling of both laparoscopic probes is almost impossible due to space restrictions.

The superior performance of the gamma-aided methods over the gold standard in this work promises further developments and suggests the evaluation of these in animal trials. The reliance of non-navigated approaches on the PET/CT images highlights a strength of the navigated approach using both modalities, namely robustness and flexibility in cases where pre-operative diagnostic images are outdated or not easily accessible, as it is often the case in the clinical setting. Moreover, due to the real-time data acquisition and visualization, dynamic changes are displayed immediately, negating the impact of deformations.

Finally, the proposed system can easily be extended to also display in the visualization therapeutic instruments such as biopsy needles, RF ablation devices and others. By including this feature the system can become a therapeutic tool.

\section{Conclusions}

A combination of navigation IOUS imaging, and nuclear labeling and detection is feasible. It will certainly be a strong triplet in the future of localization of liver metastases. Furthermore it may serve as an example toward intra-operative realtime navigation combining both functional and anatomical imaging and thus toward a therapy, in which all available information is used intelligently for the benefit of the patient.

\section{References}

1. Fong, Y., Fortner, J., Sun, R.L., Brennan, M.F., Blumgart, L.H.: Clinical score for predicting recurrence after hepatic resection for metastatic colorectal cancer: analysis of 1001 consecutive cases. Ann. Surg. 230(3), 309-321 (1999)

2. Konopke, R., Kersting, S., Bergert, H., Bloomenthal, A., Gastmeier, J., Saeger, H.D., Bunk, A.: Contrast-enhanced ultrasonography to detect liver metastases: A prospective trial to compare transcutaneous unenhanced and contrast-enhanced ultrasonography in patients undergoing laparotomy. Int. J. Colorectal Dis. 22(2), 201-207 (2007)

3. Robinson, P.J.: Imaging liver metastases: current limitations and future prospects. Br. J. Radiol. 73(867), 234-241 (2000)

4. Froehlich, A., Diederichs, C.G., Staib, L., Vogel, J., Beger, H.G., Reske, S.N.: Detection of liver metastases from pancreatic cancer using FDG PET. J. Nucl. Med. 40(2), 250-255 (1999)

5. Schillaci, O., Spanu, A., Scopinaro, F., Falchi, A., Danieli, R., Marongiu, P., Pisu, N., Madeddu, G., Fave, G.D., Madeddu, G.: Somatostatin receptor scintigraphy in liver metastasis detection from gastroenteropancreatic neuroendocrine tumors. J. Nucl. Med. 44(3), 359-368 (2003)

6. Wendler, T., Traub, J., Ziegler, S., Navab, N.: Navigated three dimensional beta probe for optimal cancer resection. In: Larsen, R., Nielsen, M., Sporring, J. (eds.) MICCAI 2006. LNCS, vol. 4190, pp. 561-569. Springer, Heidelberg (2006)

7. Prager, R., Rohling, R., Gee, A., Berman, L.: Rapid calibration for 3-d freehand ultrasound. Ultrasound in Medicine and Biology 24(6), 855-869 (1998) 
8. Lango, T.: Ultrasound Guided Surgery: Image Processing and Navigation. PhD thesis, Norwegian University of Science and Technology (2000)

9. Treece, G.M., Gee, A.H., Prager, R.W., Cash, C.J.C., Berman, L.H.: Highdefinition freehand 3-d ultrasound. Ultrasound in Medicine and Biology 29(4), 529-546 (2003)

10. Shreve, P.D., Anzai, Y., Wahl, R.L.: Pitfalls in oncologic diagnosis with FDG PET imaging: physiologic and benign variants. Radiographics 19(1), 61-77 (1999)

11. Sielhorst, T., Feuerstein, M., Traub, J., Kutter, O., Navab, N.: Campar: A software framework guaranteeing quality for medical augmented reality. International Journal of Computer Assisted Radiology and Surgery 1(suppl. 1), 29-30 (2006)

12. Bazanez-Borgert, M., Bundschuh, R.A., Herz, M., Martinez, M.J., Schwaiger, M., Ziegler, S.I.: Radioactive spheres without inactive wall for lesion simulation in pet. Z. Med. Phys. (in press)

13. Meller, B., Sommer, K., Gerl, J., von Hof, K., Surowiec, A., Richter, E., Wollenberg, B., Baehre, M.: High energy probe for detecting lymph node metastases with $18 \mathrm{~F}$ FDG in patients with head and neck cancer. Nuklearmedizin 45(4), 153-159 (2006) 